Swarthmore College

Works

$1-1-1998$

\title{
Nature Of The Blue-Phase-III-Isotropic Critical Point: An Analogy With The Liquid-Gas Transition
}

\author{
M. A. Anisimov
}

V. A. Agayan

Peter J. Collings

Swarthmore College, pcollin1@swarthmore.edu

Follow this and additional works at: https://works.swarthmore.edu/fac-physics

Part of the Physics Commons

Let us know how access to these works benefits you

\section{Recommended Citation}

M. A. Anisimov, V. A. Agayan, and Peter J. Collings. (1998). "Nature Of The Blue-Phase-III-Isotropic Critical Point: An Analogy With The Liquid-Gas Transition". Physical Review E. Volume 57, Issue 1. 582-595. DOI: 10.1103/PhysRevE.57.582

https://works.swarthmore.edu/fac-physics/156

This work is brought to you for free by Swarthmore College Libraries' Works. It has been accepted for inclusion in Physics \& Astronomy Faculty Works by an authorized administrator of Works. For more information, please contact myworks@swarthmore.edu. 


\title{
Nature of the Blue-Phase-III-isotropic critical point: An analogy with the liquid-gas transition
}

\author{
M. A. Anisimov and V. A. Agayan \\ Institute for Physical Science and Technology, University of Maryland, College Park, Maryland 20742 \\ P. J. Collings \\ Department of Physics \& Astronomy, Swarthmore College, Swarthmore, Pennsylvania 19081
}

(Received 18 August 1997)

\begin{abstract}
The analogy with the liquid-gas critical point is analyzed to clarify the nature of the pretransitional behavior of physical properties in the vicinity of the Blue-Phase-III-isotropic transition in chiral liquid crystalline systems. The analogy is unusual: temperature serves as the ordering field and entropy plays the role of the order parameter. Both mean field and parametric equations of state are formulated in terms of scaling fields. The scaling fields are linear combinations of the physical fields, which are temperature and chirality. It is shown that mixing of the physical field variables naturally leads to a strong asymmetry with respect to the transition temperature in the behavior of the physical properties that cannot be described by simple power laws. While the mean field theory gives a good description of the experimental data, the scaling theory, if one incorporates mixing of the field variables, gives even better agreement with the experimental data, placing this transition in the same universality class as the three-dimensional Ising model. [S1063-651X(97)07812-4]
\end{abstract}

PACS number(s): 64.70.Md, 65.20.+w, 61.30.-v

\section{INTRODUCTION}

Liquid crystals are phases of matter in which the molecules possess orientational (and sometimes positional) order even though the molecules undergo diffusion in much the same way as in a simple isotropic liquid [1]. In the most simple liquid crystal phase of elongated molecules, called the nematic phase, the molecules maintain a preferred direction in which to orient their long axes as they diffuse throughout the sample. If the molecules are chiral or if the liquid crystal is doped with a chiral additive, the preferred direction is no longer constant but spontaneously forms a helix. This is called the cholesteric or chiral nematic phase and the distance over which the preferred orientation rotates through $2 \pi$ radians is called the pitch. If the pitch is greater than about $500 \mathrm{~nm}$, the cholesteric phase undergoes a transition directly to the isotropic liquid phase with increasing temperature. In the case of a smaller pitch, however, the transition from the cholesteric to the isotropic liquid phase is usually via one, two, or three blue phases. These blue phases are quite exotic: (1) the preferred direction of orientation adopts a helical structure, but quite different from the structure present in the cholesteric phase, and (2) a collection of defect lines (called disclinations) form a dense network throughout the phase. In two of the blue phases, these disclinations form either a simple cubic or body centered cubic lattice. In the third blue phase $\left(\mathrm{BP}_{\mathrm{III}}\right)$, these disclinations, if they are present at all, form an amorphous structure. Keyes [2] was the first person to point out that the $\mathrm{BP}_{\mathrm{III}}$ and isotropic phases may be analogous to the liquid and gas phases, in that both have the same type of short range order and differ only quantitiatively in the degree of this order. There have been a number of recent reviews of the blue phases [3].

Recent experiments using mixtures of a pure steroisomer of a compound and the racemic mixture of the same compound have shown that the phase transition line between the amorphous blue phase $\left(\mathrm{BP}_{\mathrm{III}}\right)$ and the isotropic liquid (ISO) ends at a critical point in the temperature-chiral-fraction plane [4,5] (Fig. 1). Both heat capacity and optical measurements demonstrate that the discontinuty and the width of the two-phase region at the transition gradually disappear as the chiral fraction is increased, until one phase converts to the other continuously. This proves that the amorphous blue phase possesses macroscopically the same symmetry as the isotropic liquid. The optical properties of the two phases are slightly different, however, so below the critical point an interface is visible using a polarizing microscope [6]. A phenomenological theory based on an analogy with the liquidgas transition that introduces a new scalar order parameter suggests that the $\mathrm{BP}_{\mathrm{III}}$ transition is in the same universality class as the three-dimensional (3D) Ising model [7].

Although this phenomenological theory recognized that the physical variables, temperature and chirality, are probably not the theoretical scaling fields, nothing was done to investigate (1) how this affects the physical properties near

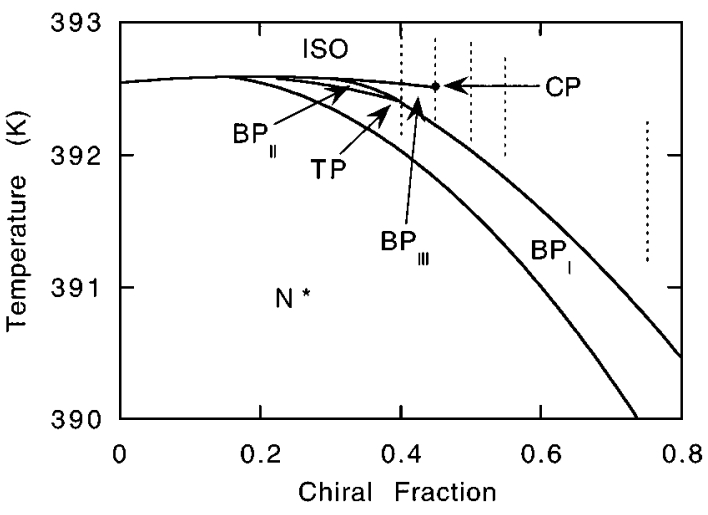

FIG. 1. $S, S$-MBBPC/rac-MBBPC phase diagram resulting from this analysis. The critical point is denoted by $\mathrm{CP}$, the Blue Phases I and II by $\mathrm{BP}_{\mathrm{I}}$ and $\mathrm{BP}_{\mathrm{II}}$, the $\mathrm{BP}_{\mathrm{I}}-\mathrm{BP}_{\mathrm{II}}-\mathrm{BP}_{\mathrm{III}}$ triple point by $\mathrm{TP}$, and the chiral nematic phase by $N^{*}$. The vertical dashed lines indicate where heat capacity data from Ref. [5] were analyzed. 
the $\mathrm{BP}_{\mathrm{III}}$-ISO transition and (2) in what ways the $\mathrm{BP}_{\mathrm{III}}-\mathrm{ISO}$ transition might be different from the liquid-gas transition. In fact, there has been extensive work on the mixing of the physical variables, temperature and chemical potentials, in near-critical one-component fluids and binary fluids [8-11]. In the simplest example of such a system, namely, a onecomponent fluid near the liquid-gas critical point, the chemical potential is one of the theoretical scaling fields (the ordering field), while the other theoretical scaling field (the nonordering field) is a mixture of temperature and chemical potential. In the liquid-gas system, however, the degree to which the chemical potential contributes to the nonordering field is very small and the lattice gas model, which does not contain mixing at all, serves as a good approximation [12].

Following the work done in the liquid-gas system [10], we first derive general expressions for the entropy and the molar heat capacity that incorporate the mixing of the physical variables. When this formulation is applied to the $\mathrm{BP}_{\mathrm{III}}$-ISO transition, it is clear that this mixing has very different consequences compared to the liquid-gas transition, in such a way that temperature serves as the ordering field and entropy plays the role of the order parameter. The molar heat capacity generally appears to be a function of three susceptibilities ("strong," "weak," and "cross"). To calculate these susceptibilities, one needs an explicit form of the equation of state. We develop two alternative equations of state, one based on the Landau expansion (mean field theory) and the other a parametric equation of state obeying scaling theory. In both cases, the large amount of mixing of the physical variables in the theoretical scaling field produces such a strong asymmetry with respect to the transition temperature that simple power laws cannot be used even very close to the critical point. The mean field equation of state agrees reasonably well with the experimental data, but the parametric equation of state obeying scaling theory gives even better agreement with the experimental data. This is firm evidence that the $\mathrm{BP}_{\mathrm{III}}-\mathrm{ISO}$ transition is in the same universality class as the 3D Ising model.

The best experimental evidence available is the heat capacity data for a near-critical chiral-racemic mixture of $S, S$-(+)-4' '-(methylbutyl) phenyl-4'-(methylbutyl) biphenyl carboxylate ( $S, S$-MBBPC) [5]. These data are analyzed using both equations of state to find the coefficients that describe the mixing of the physical variables. These values are then used to show that the heat capacity data from other regions of the phase diagram are also accurately described. Since there are also optical activity and turbidity results for this system, it is also shown that the same coefficients describe these data if the optical properties are assumed to depend on a linear combination of the two scaling densities.

Finally, the pure compound $S$-(+)-4-(2-methylbutyl) phenyl-4-hexyoxybenzoate ( $S$-CE4) is also near the critical point, and latent heat experiments have been performed on mixtures of this compound with its racemic mixture [13]. The latent heat, optical activity, and light scattering data are analyzed using both equations of state to find the mixing coefficients for this system. Both mean field theory and scaling theory describe the data quite well. Although these data alone do not support one theory over the other, they show that a consistent selection of parameters enables one to de- scribe both the thermodynamic and optical properties in this system also.

\section{SCALING FIELDS AND MIXING OF PHYSICAL FIELDS}

We consider a system with an isolated critical point that is characterized by two relevant scaling fields, an ordering field $h_{1}$ conjugate to the order parameter $\varphi_{1}$, and a nonordering field $h_{2}$ conjugate to the second scaling density $\varphi_{2}[14,15]$. It is well established that fluids, fluid mixtures, and binary alloys belong to the same universality class as the 3D Ising model (or, equivalently, the lattice gas) [9,12,16-20].

Near the critical point the singular part of the fielddependent dimensionless density of a relevant thermodynamic potential $(\Phi)$ satisfies the following scaling law:

$$
\Delta \widetilde{\Phi}\left(h_{1}, h_{2}\right)=\Delta \Phi / V \rho_{\mathrm{c}} R T_{\mathrm{c}}=h_{2}^{2}\left|h_{2}\right|^{-\alpha} f(z),
$$

with

$$
z=h_{1} /\left|h_{2}\right|^{\beta+\gamma},
$$

where $f(z)$ is a universal scaling function with known asymptotic behavior, $T_{\mathrm{c}}$ is the critical temperature, $\rho_{\mathrm{c}}$ is the critical density, $V$ is the volume, and $\alpha, \beta$, and $\gamma$ are universal critical exponents interrelated by the relationship $\alpha$ $+2 \beta+\gamma=2$. For the universality class of the 3D Ising model, $\alpha \simeq 0.11, \beta \simeq 0.325$, and $\gamma \simeq 1.24$ [12,21]. The relevant thermodynamic potential for fluids is the grand thermodynamic potential $\Omega=-P V$, where $P$ is the pressure. As liquid crystals are weakly compressible systems, an appropriate choice for the thermodynamic potential is the Gibbs free energy.

The scaling "densities" conjugate to $h_{1}$ and $h_{2}$ are

$$
\begin{gathered}
\varphi_{1}=-\left(\frac{\partial \Delta \widetilde{\Phi}}{\partial h_{1}}\right)_{h_{2}}=\left|h_{2}\right|^{\beta} f^{\prime}(z), \\
\varphi_{2}=-\left(\frac{\partial \Delta \widetilde{\Phi}}{\partial h_{2}}\right)_{h_{1}}=h_{2}\left|h_{2}\right|^{-\alpha} \psi(z),
\end{gathered}
$$

where $f^{\prime}(z)=d f / d z$ and

$$
\psi(z)=(2-\alpha) f(z)-(\beta+\gamma) z f^{\prime}(z) .
$$

One may define the susceptibilities $\chi_{1}$ (strongly divergent) and $\chi_{2}$ (weakly divergent), which are associated with the densities $\varphi_{1}$ and $\varphi_{2}$ :

$$
\begin{aligned}
& \chi_{1}=\left(\frac{\partial \varphi_{1}}{\partial h_{1}}\right)_{h_{2}}=\left|h_{2}\right|^{-\gamma} f^{\prime \prime}(z), \\
& \chi_{2}=\left(\frac{\partial \varphi_{2}}{\partial h_{2}}\right)_{h_{1}}=\left|h_{2}\right|^{-\alpha} \Psi(z),
\end{aligned}
$$

with $\Psi(z)=(1-\alpha) \psi(z)-(\beta+\gamma) z \psi^{\prime}(z)$ and where $f^{\prime \prime}(z)$ $=d^{2} f / d z^{2}$ and $\psi^{\prime}(z)=d \psi / d z$. In addition one may define a cross susceptibility as 


$$
\begin{aligned}
\chi_{12} & =\chi_{21}=\left(\frac{\partial \varphi_{1}}{\partial h_{2}}\right)_{h_{1}}=\left(\frac{\partial \varphi_{2}}{\partial h_{1}}\right)_{h_{2}} \\
& =\left|h_{2}\right|^{\beta-1}\left[\beta f^{\prime}(z)-(\beta+\gamma) z f^{\prime \prime}(z)\right] .
\end{aligned}
$$

Specifically, in zero ordering field $\left(h_{1}=0\right)$, the scaling densities and the scaling susceptibilities behave as $\varphi_{1}=$ $\pm B_{0}\left|h_{2}\right|^{\beta}, \varphi_{2}=\left[A_{0}^{-} /(1-\alpha)\right] h_{2}\left|h_{2}\right|^{-\alpha}$, and $\chi_{1}=\Gamma_{0}^{ \pm}\left|h_{2}\right|^{-\gamma}$, $\chi_{2}=A_{0}^{ \pm}\left|h_{2}\right|^{-\alpha}$ with system-dependent scaling amplitudes $A_{0}^{ \pm}=(2-\alpha)(1-\alpha) f_{ \pm}(0), B_{0}=f_{-}^{\prime}(0)$, and $\Gamma_{0}^{ \pm}=f_{+}^{\prime \prime}(0)$, where $A_{0}^{ \pm}, B_{0}$, and $\Gamma_{0}^{ \pm}$are system-dependent amplitudes interrelated by the universal amplitude ratios $\alpha A_{0}^{+} \Gamma_{0}^{+} / B_{0}^{2}$ $\simeq 0.06, \Gamma^{+} \simeq 4.9 \Gamma^{-}$, and $A_{0}^{-} \simeq 1.9 A_{0}^{+}$, so that only two of the amplitudes are independent [17]. The superscripts or subscripts "plus" and "minus" correspond to limits of $f(z)$ taken above $\left(h_{2}>0\right)$ and below $\left(h_{2}<0\right)$ the critical point, respectively. We note that above the critical point in zero ordering field $\varphi_{1}=0, \varphi_{2}=0$, and $\chi_{12}=0$ since $z=0$ and $f^{\prime}(z)=0$.

The simple Ising model and the lattice gas model have a special symmetry with respect to the sign of the ordering field. For the Ising model, the ordering field is the magnetic field, and the order parameter is the magnetization [19]. For the lattice gas, since the relevant thermodynamic potential is $\Omega=-P V$ and its density is the negative of the pressure $P$, the chemical potential difference $g-g_{\text {c }}$ plays the role of the ordering field, and the density difference $\rho-\rho_{\mathrm{c}}$ is the order parameter [17]:

$$
\begin{gathered}
h_{1}=\left(g-g_{\mathrm{c}}\right) / R T_{\mathrm{c}}=\Delta g, \\
\varphi_{1}=\left(\rho-\rho_{\mathrm{c}}\right) / \rho_{\mathrm{c}}=\Delta \rho .
\end{gathered}
$$

The nonordering field $h_{2}$ and its conjugate scaling density $\varphi_{2}$ are

$$
\begin{gathered}
h_{2}=\left(T-T_{\mathrm{c}}\right) / T_{\mathrm{c}}=\Delta T, \\
\varphi_{2}=\left(\rho S-\rho_{\mathrm{c}} S_{\mathrm{c}}\right) / \rho_{\mathrm{c}} R=\Delta S,
\end{gathered}
$$

where $S$ is the molar entropy and $R$ the universal gas constant.

Real fluids near the liquid-gas critical point, however, do not possess the symmetry of the lattice gas. The physical fields, which are the chemical potential and temperature, have no definite scaling dimensionality and one should identify the scaling fields with the linear combinations $[9,10]$ :

$$
\begin{aligned}
& h_{1}=a_{1} \Delta g+a_{2} \Delta T, \\
& h_{2}=b_{1} \Delta T+b_{2} \Delta g,
\end{aligned}
$$

where $a_{i}$ and $b_{i}$ are system-dependent coefficients. The scaling fields may be normalized in such a way that $a_{1}=1$ and $b_{1}=1$, but then two system-dependent critical amplitudes appear (for instance, $\Gamma_{0}^{+}$and $B_{0}$ ).

Near the liquid-gas critical point, the coefficient $a_{2}$ depends on the choice of zero entropy and is arbitrary in classical thermodynamics, i.e., can be chosen to be zero without affecting any measurable properties. As soon as the value of $a_{2}$ is assigned, the value of $b_{2}$, which is often referred to as the mixing parameter $[8,12]$, becomes well defined $[10,11]$. In simple fluids the magnitude of the mixing parameter is small $\left(b_{2}<0.1\right)$ [22], and the main contribution to the scaling field $h_{2}$ comes from the temperature.

Taking $a_{2}=0$ corresponds to the choice of the critical point entropy $S_{\mathrm{c}}=\rho_{\mathrm{c}}^{-1}(\partial P / \partial T)_{\rho_{\mathrm{c}}}$, and we have for the scaling fields and for the conjugate densities

$$
\begin{gathered}
h_{1}=\Delta g, \\
h_{2}=\Delta T+b_{2} h_{1}, \\
\varphi_{1}=\Delta \rho-b_{2} \Delta S, \\
\varphi_{2}=\Delta S .
\end{gathered}
$$

The physical response functions, namely, the isothermal compressibility and the isochoric heat capacity $\left(C_{V} / R\right)$, can be expressed through the scaling susceptibilities (the superscript " $r$ " denotes the regular part of a physical quantity)

$$
\begin{gathered}
\rho\left(\frac{\partial \rho}{\partial p}\right)_{T}=\rho\left(\frac{\partial \rho}{\partial p}\right)_{T}^{\mathrm{r}}+\rho_{\mathrm{c}} R T_{\mathrm{c}}\left(\chi_{1}+b_{2}^{2} \chi_{2}+2 b_{2} \chi_{12}\right) \\
\left(\rho / \rho_{\mathrm{c}}\right)\left(C_{V} / R\right)=\frac{T}{\rho_{\mathrm{c}} R}\left(\frac{\partial S}{\partial T}\right)_{\rho}^{\mathrm{r}}+\frac{b_{1}^{2} \chi_{2}}{1+b_{2}^{2} \chi_{2} \chi_{1}^{-1}} .
\end{gathered}
$$

One can see that the main contribution to the compressibility of a near critical fluid is the strongly divergent susceptibility $\chi_{1}$, while the main contribution to the isochoric heat capacity is the weakly divergent susceptibility $\chi_{2}$. As

$$
C_{P}-C_{V}=\frac{T}{\rho^{2}}\left(\frac{\partial P}{\partial T}\right)_{\rho}\left(\frac{\partial \rho}{\partial P}\right)_{T},
$$

the molar heat capacity at constant pressure asymptotically behaves as the isothermal compressibibility.

In liquid crystals near the $\mathrm{BP}_{\mathrm{III}}$-ISO transition, the physical meanings of the scaling fields and scaling densities are very different. The physical fields at constant pressure in this case are the temperature and chirality [7]. The best definition of chirality is probably the coefficient in front of the chiral term in the thermodynamic potential [7], but this is never measured directly. In practice, the chirality $\kappa$ can be tuned by the average (overall) composition $X$ of a mixture of a highly chiral compound with its racemic mixture (chiral fraction), so that the density of the Gibbs free energy is

$$
\widetilde{\Phi}[P, T, \kappa(X)]=\widetilde{\Phi}\left(P, h_{1}, h_{2}\right)+\widetilde{\Phi}^{\mathrm{r}}(P, T, X) .
$$

Thus the scaling fields are linear combinations of the temperature and chiral fraction $[5,7]$

$$
\begin{aligned}
& h_{1}=a_{1} \Delta X+a_{2} \Delta T, \\
& h_{2}=b_{1} \Delta T+b_{2} \Delta X,
\end{aligned}
$$

where $\Delta X=\left(X-X_{\mathrm{c}}\right) / X_{\mathrm{c}}$, and $X_{\mathrm{c}}$ is the critical chiral fraction. In contrast to a liquid-gas critical point, all the coefficients $a_{i}$ and $b_{i}$ are well defined. The ratio $a_{1} / a_{2}$ can be found experimentally from the slope $\Delta T / \Delta X$ of the phase 
transition line $\left(h_{1}=0\right)$. All currently available experimental results show this slope to be very small. Figure 1 indicates that the slope may be slightly negative in the $S, S$-MBBPC system [5]. Therefore, the ordering field $h_{1}$ is associated mainly with temperature. We also note that the coefficient $a_{2}$ must be negative for the $\mathrm{BP}_{\mathrm{III}}$-ISO transition, as higher temperature corresponds to the state with lower ordering.

Inverting Eqs. (23) and (24) we can express $\Delta T$ and $\Delta X$ in terms of $h_{1}$ and $h_{2}$ as

$$
\begin{aligned}
& \Delta T=\left(\frac{b_{2}}{a_{2} b_{2}-b_{1} a_{1}}\right) h_{1}+\left(\frac{a_{1}}{b_{1} a_{1}-a_{2} b_{2}}\right) h_{2}, \\
& \Delta X=\left(\frac{a_{2}}{a_{2} b_{2}-b_{1} a_{1}}\right) h_{2}+\left(\frac{b_{1}}{b_{1} a_{1}-a_{2} b_{2}}\right) h_{1} .
\end{aligned}
$$

The entropy enhancement $\Delta S$ is a linear combination of the two scaling densities $\varphi_{1}$ and $\varphi_{2}$, conjugate to the scaling fields $h_{1}$ and $h_{2}$, respectively:

$$
\Delta S=-\left(\frac{\partial \Delta \Phi}{\partial \Delta T}\right)_{P, X}=a_{2} \varphi_{1}+b_{1} \varphi_{2}
$$

Note, as $\varphi_{1} \propto\left|h_{1}\right|^{\beta}, \varphi_{2} \propto\left|h_{1}\right|^{1-\alpha}$, and as $\beta<1-\alpha$, the leading term in the entropy is associated with the order parameter $\varphi_{1}$.

One may introduce a densitylike variable $\zeta$ conjugate to the chiral fraction. As this variable is a thermodynamic coordinate, one can reasonably assume it to be proportional to the inverse pitch. Like the entropy, the enhancement in this variable, $\Delta \zeta$, is a linear combination of the two scaling densities:

$$
\Delta \zeta=a_{1} \varphi_{1}+b_{2} \varphi_{2} .
$$

If $a_{1}$ is very small, the contribution to $\Delta \zeta$ from $b_{2} \varphi_{2}$ dominates. For $\varphi_{1}$ and $\varphi_{2}$ we then find

$$
\begin{aligned}
& \varphi_{1}=\left(\frac{b_{1}}{a_{1} b_{1}-a_{2} b_{2}}\right) \Delta \zeta-\left(\frac{b_{2}}{a_{1} b_{1}-a_{2} b_{2}}\right) \Delta S, \\
& \varphi_{2}=\left(\frac{a_{1}}{a_{1} b_{1}-a_{2} b_{2}}\right) \Delta S-\left(\frac{a_{2}}{a_{1} b_{1}-a_{2} b_{2}}\right) \Delta \zeta .
\end{aligned}
$$

The singular part of the isobaric heat capacity $C_{P, X}$ is expressed in terms of the susceptibilities:

$$
\begin{aligned}
C_{P, X}-C_{P, X}^{\mathrm{r}} & =R T\left(\frac{\partial \Delta S}{\partial T}\right)_{P, X}=a_{2} \frac{R T}{T_{\mathrm{c}}}\left(\frac{\partial \Delta S}{\partial h_{1}}\right)_{P, X} \\
& =a_{2} \frac{R T}{T_{\mathrm{c}}}\left[\left(\frac{\partial \Delta S}{\partial h_{1}}\right)_{h_{2}, X}+\left(\frac{\partial \Delta S}{\partial h_{2}}\right)_{h_{1}, X}\left(\frac{\partial h_{2}}{\partial h_{1}}\right)_{P, X}\right] \\
& =a_{2} \frac{R T}{T_{\mathrm{c}}}\left[a_{2}\left(\frac{\partial \varphi_{1}}{\partial h_{1}}\right)_{h_{2}}+b_{1}\left(\frac{\partial \varphi_{2}}{\partial h_{1}}\right)_{h_{2}}\right]+a_{2} \frac{R T}{T_{\mathrm{c}}}\left[a_{2}\left(\frac{\partial \varphi_{1}}{\partial h_{2}}\right)_{h_{1}}+b_{1}\left(\frac{\partial \varphi_{2}}{\partial h_{2}}\right)_{h_{1}}\right]_{\frac{b_{1}}{a_{2}}} \\
& =\frac{R T}{T_{\mathrm{c}}}\left(a_{2}^{2} \chi_{1}+b_{1}^{2} \chi_{2}+2 a_{2} b_{1} \chi_{12}\right) .
\end{aligned}
$$

Note that near the critical point $\chi_{1} \gg\left|\chi_{12}\right| \gg \chi_{2}$. Therefore, the leading term in Eq. (31) is the strongly divergent susceptibility $\chi_{1}$.

We emphasize the essential difference between the physical meanings of the scaling fields and scaling densities for the liquid-gas phase transition on the one hand, and the $\mathrm{BP}_{\mathrm{III}}$-ISO transition on the other hand (see Table I). In fluids near the liquid-gas critical point, the strongly divergent susceptibility $\chi_{1}$ is mainly associated with the isothermal compressibility, while the isochoric heat capacity serves as the weakly divergent susceptibility $\chi_{2}$. The order parameter $\varphi_{1}$ is mainly associated with the molar density and $\varphi_{2}$ with the molar entropy only. The mixing of the physical fields does not change the asymptotic critical behavior but causes nonasymptotic corrections like the so-called "singular diameter" of the liquid-gas coexistence curve [23]:

$$
\frac{\rho_{L}+\rho_{V}}{2 \rho_{\mathrm{c}}}=b_{2} \varphi_{2}=b_{2} \frac{A_{0}^{-}}{1-\alpha} h_{2}\left|h_{2}\right|^{-\alpha},
$$

where $\rho_{L}$ and $\rho_{V}$ are the liquid and gas densities, respectively.

The $\mathrm{BP}_{\mathrm{III}}$ criticality is different. The actual density here is an irrelevant variable as it is the same in the coexisting phases and does not change much with temperature. The ordering field is associated mainly with temperature. The isobaric heat capacity approximately serves as the strongly divergent susceptibility $\chi_{1}$, although the more weakly divergent susceptibilities, $\chi_{12}$ and $\chi_{2}$, also contribute to $C_{P, X}$ when the distance from the critical point increases. The order parameter is mainly associated with the entropy, so that measurements of the latent heat as a function of chiral fraction approaching the critical point could give direct information on the behavior of the order parameter.

\section{MEAN FIELD (CLASSICAL) THEORY}

It is speculated [5] that the $\mathrm{BP}_{\mathrm{III}}$-ISO transition exhibits mean field criticality. That is why we will first consider mean 
TABLE I. "Cartoon" analogy between the liquid-gas and $\mathrm{BP}_{\mathrm{III}}$-ISO criticalities.

\begin{tabular}{|c|c|c|}
\hline & \multicolumn{2}{|c|}{ Leading contributions } \\
\hline & Liquid-gas & $\mathrm{BP}_{\mathrm{III}}-\mathrm{ISO}$ \\
\hline $\begin{array}{l}\text { Order parameter } \\
\text { (first scaling density) } \\
\varphi_{1} \text { conjugate to } h_{1}\end{array}$ & Density & Entropy \\
\hline $\begin{array}{l}\text { Ordering scaling } \\
\text { field } h_{1}\end{array}$ & $\begin{array}{l}\text { Chemical } \\
\text { potential }\end{array}$ & Temperature \\
\hline $\begin{array}{l}\text { Nonordering } \\
\text { scaling field } h_{2}\end{array}$ & Temperature & $\begin{array}{l}\text { Temperature mixed } \\
\text { with chirality }\end{array}$ \\
\hline $\begin{array}{l}\text { Second scaling } \\
\text { density } \varphi_{2} \\
\text { conjugate to } h_{2}\end{array}$ & Entropy & Inverse pitch \\
\hline $\begin{array}{l}\text { Strong susceptibility } \\
\chi_{1}=\left(\partial \varphi_{1} / \partial h_{1}\right)_{h_{2}}\end{array}$ & $\begin{array}{c}\text { Isothermal } \\
\text { compressibility }\end{array}$ & $\begin{array}{l}\text { Isobaric heat capacity } \\
\text { at constant chirality }\end{array}$ \\
\hline $\begin{array}{l}\text { Weak susceptibility } \\
\chi_{2}=\left(\partial \varphi_{2} / \partial h_{2}\right) h_{1}\end{array}$ & $\begin{array}{c}\text { Isochoric } \\
\text { heat capacity }\end{array}$ & $\begin{array}{c}\text { Combination of } \\
\text { temperature and } \\
\text { chirality derivatives } \\
\text { of the pitch }\end{array}$ \\
\hline
\end{tabular}

field (classical) theory in which the fluctuations of the order parameter are neglected.

The critical part of the classical field-dependent thermodynamic potential at constant pressure $\Delta \widetilde{\Phi}\left(P, h_{1}, h_{2}\right)$, which is again a function of two scaling fields $h_{1}$ and $h_{2}$, is given by the Landau expansion in powers of the order parameter $\varphi_{1}[9,20]$,

$$
\Delta \widetilde{\Phi}\left(h_{1}, h_{2}\right)=\frac{1}{2} a_{0} h_{2} \varphi_{1}^{2}+\frac{1}{4} u_{0} \varphi_{1}^{4}-h_{1} \varphi_{1},
$$

where the constants $a_{0}$ and $u_{0}$ play the role of the classical system-dependent amplitudes. Minimization of this thermodynamic potential with respect to $\varphi_{1}$ yields the following equation, which defines the equilibrium value of the order parameter:

$$
u_{0} \varphi_{1}^{3}+a_{0} h_{2} \varphi_{1}-h_{1}=0 .
$$

Using Eq. (34) we find expressions for $\varphi_{2}$ and susceptibilities $\chi_{1}, \chi_{2}$, and $\chi_{12}$ :

$$
\begin{gathered}
\varphi_{2}=-\left(\frac{\partial \Delta \widetilde{\Phi}}{\partial h_{2}}\right)_{h_{1}}=-\frac{1}{2} a_{0} \varphi_{1}^{2} \\
\chi_{1}=\left(\frac{\partial h_{1}}{\partial \varphi_{1}}\right)_{h_{2}}^{-1}=\left(a_{0} h_{2}+3 u_{0} \varphi_{1}^{2}\right)^{-1} \\
\chi_{12}=\left(\frac{\partial h_{2}}{\partial \varphi_{1}}\right)_{h_{1}}^{-1}=-a_{0} \varphi_{1} /\left(a_{0} h_{2}+3 u_{0} \varphi_{1}^{2}\right)=-a_{0} \varphi_{1} \chi_{1} \\
\chi_{2}=\left(\frac{\partial h_{2}}{\partial \varphi_{2}}\right)_{h_{1}}^{-1}=-a_{0} \varphi_{1}\left(\frac{\partial h_{2}}{\partial \varphi_{1}}\right)_{h_{1}}^{-1}=a_{0}^{2} \varphi_{1}^{2} \chi_{1}
\end{gathered}
$$

The critical part of the entropy is

$$
\Delta S=a_{2} \varphi_{1}+b_{1} \varphi_{2}=a_{2} \varphi_{1}-\frac{b_{1} a_{0}}{2} \varphi_{1}^{2} .
$$

The isobaric heat capacity in classical theory is

$$
C_{P, X}=R T\left(a_{2}-a_{0} b_{1} \varphi_{1}\right)\left(\frac{\partial \varphi_{1}}{\partial T}\right)_{P, X}+C_{P, X}^{\mathrm{r}} .
$$

Since

$$
\left(\frac{\partial \varphi_{1}}{\partial T}\right)_{P, X}=\left(\frac{\partial \varphi_{1}}{\partial h_{2}}\right)_{P, h_{1}}\left(\frac{\partial h_{2}}{\partial T}\right)_{P, X}+\left(\frac{\partial \varphi_{1}}{\partial h_{1}}\right)_{P, h_{2}}\left(\frac{\partial h_{1}}{\partial T}\right)_{P, X},
$$

one obtains

$$
\left(\frac{\partial \varphi_{1}}{\partial T}\right)_{P, X}=\frac{1}{T_{\mathrm{c}}} \frac{a_{2}-a_{0} b_{1} \varphi_{1}}{a_{0} h_{2}+3 u_{0} \varphi_{1}^{2}}
$$

and, finally,

$$
\begin{aligned}
C_{P, X} & =\frac{R T}{T_{\mathrm{c}}} \frac{\left(a_{2}-a_{0} b_{1} \varphi_{1}\right)^{2}}{a_{0} h_{2}+3 u_{0} \varphi_{1}^{2}}+C_{P, X}^{\mathrm{r}} \\
& =a_{2}^{2} \chi_{1} \frac{R T}{T_{\mathrm{c}}}\left(1-\frac{a_{0} b_{1}}{a_{2}} \varphi_{1}\right)^{2}+C_{P, X}^{\mathrm{r}},
\end{aligned}
$$

which satisfies the general expression (31) taking Eqs. (36)(38) into account. Graphs of the mean field scaling densities and susceptibilities using parameters appropriate for the systems to be studied are shown in Fig. 2.

\section{SCALING (LINEAR MODEL) EQUATION OF STATE}

The expressions for the scaling function $f(z)$ and its derivatives can be found in some approximation by renormalization group theory, but the calculations are cumbersome and inconvenient for practical use [20]. This is why a parametric representation of the equation of state near the critical point [24-27] has become very popular.

The simplest form of the parametric equations of state is the so-called "linear model" with the "polar" variables $r$ and $\theta[24,28]$,

$$
\begin{gathered}
h_{1}=a r^{\beta+\gamma} \theta\left(1-\theta^{2}\right), \\
h_{2}=r\left(1-b^{2} \theta^{2}\right), \\
\varphi_{1}=k r^{\beta} \theta,
\end{gathered}
$$

where the parameter $r$ represents the distance to the critical point, the parameter $\theta$ is the distance along a contour of constant $r$ (see Fig. 3), the coefficient $b^{2}=(\gamma-2 \beta) / \gamma(1$ $-2 \beta$ ) is a universal constant, and $a$ and $k$ are systemdependent constants.

The singular behavior of thermodynamic functions is defined by the variable $r$, whereas the thermodynamic quantities are analytic functions with respect to $\theta$. This parametric form of the equation of state satisfies the power-law behavior of the thermodynamic functions that follows from scaling 


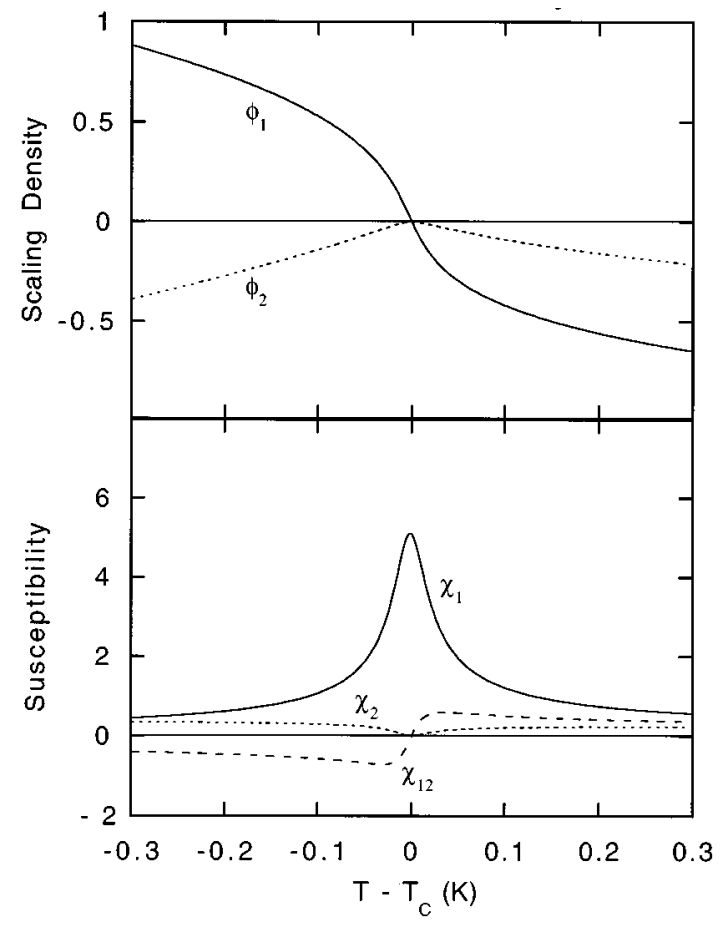

FIG. 2. Mean field theory "densities" $\varphi_{1}$ and $\varphi_{2}$ and susceptibilities $\chi_{1}, \chi_{2}$, and $\chi_{12}$ calculated with the parameters from the $X$ $=0.45$ heat capacity fit.

theory. The equation of state expressed through the traditional variables $h_{1}, h_{2}$, and $\varphi_{1}$ is determined by eliminating $r$ and $\theta$ from the above equations.

The singular part of the thermodynamic potential is given by [17]

$$
\begin{aligned}
\Delta \widetilde{\Phi(r, \theta)=} & a k r^{2-\alpha}\left[f(\theta)-\theta^{2}\left(1-\theta^{2}\right)\right] \\
& +(a k / 6) r^{2}\left(1-b^{2} \theta^{2}\right)^{2}
\end{aligned}
$$

which we modified by adding the last analytic term to make the model fully consistent with the results of renormalization group (RG) theory. According to RG theory, there is a fluctuation-induced analytic term in expressions for the entropy and heat capacity [29]. The corresponding term in the thermodynamic potential $\Delta \widetilde{\Phi}\left(h_{1}, h_{2}\right)$ is proportional to $h_{2}^{2}$ and is known as the kernel term [29]. The second scaling density $\varphi_{2}$ is obtained by differentiation of Eq. (47), yielding

$$
\varphi_{2}=a k r^{1-\alpha} s(\theta)-a k r\left(1-b^{2} \theta^{2}\right) / 3 .
$$

Using the definitions of the susceptibilities (6)-(8) one finds

$$
\begin{gathered}
\chi_{1}=(k / a) r^{-\gamma} c_{1}(\theta), \\
\chi_{12}=k r^{\beta-1} c_{12}(\theta), \\
\chi_{2}=a k r^{-\alpha} c_{2}(\theta)-a k / 3 .
\end{gathered}
$$

In Eqs. (48)-(51) $f(\theta), s(\theta)$, and $c_{i}(\theta)$ are known functions of $\theta$ :

$$
f(\theta)=f_{0}+f_{2} \theta^{2}+f_{4} \theta^{4},
$$

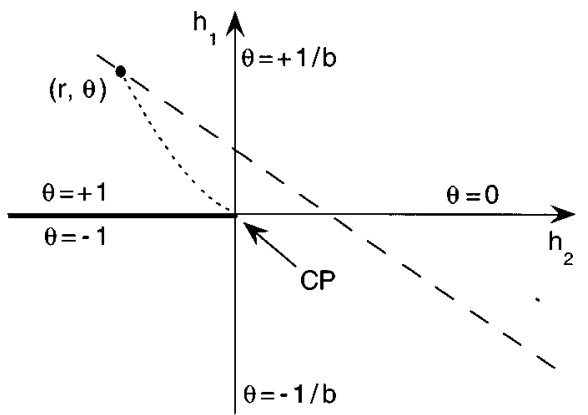

FIG. 3. "Polar" variables $r$ and $\theta$ used in the parametric representation of the equation of state. The critical point is denoted by $\mathrm{CP}$ and the bold line is the first order transition line. The point labeled $(r, \theta)$ represents an arbitrary starting point for a constant $\theta$ path through the critical point (dotted line) and a constant $\Delta X$ path that misses the critical point (dashed line).

$$
\begin{gathered}
f_{0}=-\frac{\beta(\delta-3)-b^{2} \alpha \gamma}{2 b^{4}(2-\alpha)(1-\alpha) \alpha}, \\
f_{2}=\frac{\beta(\delta-3)-b^{2} \alpha(1-2 \beta)}{2 b^{2}(1-\alpha) \alpha} \\
f_{4}=-\frac{1-2 \beta}{2 \alpha}, \\
s(\theta)=s_{0}+s_{2} \theta^{2}, \\
s_{0}=-(2-\alpha) f_{0}, \\
s_{2}=-(2-\alpha) b^{2}(1-2 \beta) f_{0}-\gamma f_{2}, \\
c_{1}(\theta)=\left(1-b^{2} \theta^{2}(1-2 \beta)\right) / c_{0}(\theta), \\
c_{12}(\theta)=\beta \theta\left[1-\delta-\theta^{2}(3-\delta)\right] / c_{0}(\theta), \\
c_{0}(\theta)=\left(1-3 \theta^{2}\right)\left(1-b^{2} \theta^{2}\right)+2 \beta \delta b^{2} \theta^{2}\left(1-\theta^{2}\right),
\end{gathered}
$$

and $\delta$ is the universal critical exponent, which can be found from the relation $(\delta-1) \beta=\gamma$. One can verify that these expressions satisfy the asymptotic scaling laws introduced above.

Substituting the 3D Ising values of the critical exponents into Eqs. (52)-(62), we obtain

$$
\begin{gathered}
b^{2} \simeq 1.35944, \\
f(\theta) \simeq-0.59156+2.01992 \theta^{2}-1.59091 \theta^{4}, \\
s(\theta) \simeq 1.11804-1.97273 \theta^{2}, \\
c_{0}(\theta) \simeq 1-0.10438 \theta^{2}-0.17673 \theta^{4}, \\
c_{1}(\theta) \simeq\left(1-0.47581 \theta^{2}\right) / c_{0}(\theta),
\end{gathered}
$$






FIG. 4. Scaling theory "densities" $\varphi_{1}$ and $\varphi_{2}$ and susceptibilities $\chi_{1}, \chi_{2}$, and $\chi_{12}$ calculated with the parameters from the $X$ $=0.45$ heat capacity fit.

$$
\begin{gathered}
c_{12}(\theta) \simeq \theta\left(-1.24+0.59 \theta^{2}\right) / c_{0}(\theta), \\
c_{2}(\theta)=\left(0.99506+1.43374 \theta^{2}-0.90745 \theta^{4}\right) / c_{0}(\theta) .
\end{gathered}
$$

Graphs of the scaling densities and susceptibilities using parameters appropriate to the systems to be studied are shown in Fig. 4.

The linear model also yields the following expressions for the critical amplitudes:

$$
\begin{gathered}
A_{0}^{+}=\frac{a k \gamma(\gamma-1)}{2 b^{2} \alpha}, \\
B_{0}=\frac{k}{\left(b^{2}-1\right)^{\beta}}, \\
\Gamma_{0}^{+}=\frac{k}{a} .
\end{gathered}
$$

Now we will show that the results of mean field (classical) theory can be expressed through the parametric variables as well. The magnitudes of the critical exponents in mean field theory are $\alpha=0, \beta=1 / 2, \gamma=1, \delta=3$. To find the value of the universal constant $b^{2}=(\gamma-2 \beta) / \gamma(1-2 \beta)$ in mean field theory, one has to employ the $\epsilon$ expansion for the critical exponents [30,31]. The parameter $\epsilon$ is related to the dimensionality $d$ of space by $\epsilon=4-d$. The mean field results are valid in the limit $\epsilon \rightarrow 0$. In first order of $\epsilon$, the expansion gives $2 \beta=1-\frac{1}{3} \epsilon, \gamma=1+\frac{1}{6} \epsilon, \alpha=\frac{1}{6} \epsilon$, thus $b^{2}=3 / 2$.

Substituting the classical values of the critical exponents and $b^{2}$ into Eqs. (52)-(62), we obtain

$$
\begin{gathered}
f(\theta)=-1 / 6+\theta^{2}-\theta^{4}, \quad s(\theta)=1 / 3-\theta^{2}, \\
c_{0}(\theta)=1, \quad c_{1}(\theta)=1, \quad c_{12}(\theta)=-\theta, \quad c_{2}(\theta)=1 / 3+\theta^{2},
\end{gathered}
$$

and for the scaling densities and susceptibilities in the parametric form:

$$
\begin{gathered}
\varphi_{1}=k r^{1 / 2} \theta, \\
\varphi_{2}=-\frac{1}{2} a k r \theta^{2}=-\frac{1}{2} a_{0} \varphi_{1}^{2}, \\
\chi_{1}=(k / a) r^{-1}=\left[a_{0} h_{2}+3 u_{0} \varphi_{1}^{2}\right]^{-1}, \\
\chi_{12}=-k r^{-1 / 2} \theta=-a_{0} \varphi_{1} \chi_{1}, \\
\chi_{2}=a k \theta^{2}=\frac{a_{0}^{2}}{2 u_{0}} \theta^{2}=a_{0}^{2} \varphi_{1}^{2} \chi_{1},
\end{gathered}
$$

where the coefficients $a_{0}$ and $u_{0}$ are related to $k$ and $a$ as follows:

$$
\begin{gathered}
a_{0}=a / k, \\
u_{0}=a / 2 k^{3}, \\
a k=a_{0}^{2} / 2 u_{0} .
\end{gathered}
$$

\section{INTERPRETATION OF THE EXPERIMENTAL DATA}

\section{A. Isobaric heat capacity}

The isobaric heat capacity should be fitted to Eq. (31) with the susceptibilities taken either from mean field theory [Eqs. (36) -(38)] or with use of the scaling parametric equations (49)-(51). A more general approach is to use a crossover theory that incorporates both the Ising asymptotic critical behavior and possible mean field behavior far away from the critical point [29]. However, such a fit requires more accurate data and is more sophisticated, since the crossover functions are not explicit and at least one additional adjustable parameter, namely, a crossover scale (Ginzburg number), appears [29].

One should note that the structure and the meaning of Eq. (31) are substantially different from the expression

$$
C_{P, X}=A_{1}^{ \pm}|\Delta T|^{-x}+A_{2}^{ \pm}|\Delta T|^{-y}+B^{ \pm}+E(T),
$$

used for fitting the experimental data in [5]. In this equation $x$ and $y$ are adjustable exponents. The superscripts " + "' and " - " correspond to the phases above and below $T_{\mathrm{c}}$, respectively. Even along the path $X=X_{\mathrm{c}}$, where the scaling fields, the order parameter, and the heat capacity are functions of temperature only, none of the susceptibilities $\chi_{1}, \chi_{2}$, or $\chi_{12}$, entering Eq. (31) obeys the simple power-law structure of Eq. (83)

\section{B. Latent heat}

The latent heat $L$ is defined as

$$
L=T_{B I}\left(S_{I}-S_{B}\right)
$$


where $T_{B I}$ is the $\mathrm{BP}_{\mathrm{III}}$-ISO transition temperature, $S_{I}$ is the entropy of the isotropic liquid phase, and $S_{B}$ is the entropy of $\mathrm{BP}_{\mathrm{III}}$. Since the order parameter $\varphi_{1}$ is equal to but of opposite sign on either side of the transition, while the second scaling density $\varphi_{2}$ is the same on either side of the transition, Eq. (27) yields the following simple expression for the latent heat:

$$
L / R T_{B I}=2\left|a_{2} \varphi_{1}\right|
$$

with $\varphi_{1}$ taken either from Eq. (34) (mean field theory) or from Eq. (46) (scaling theory).

\section{Optical activity and light scattering}

The optical activity and intensity of light scattering, being densitylike quantities (different in the coexisting phases), should have a component depending on $\Delta \zeta$, conjugate to the chiral fraction $\Delta X$. Therefore, like entropy, the optical activity $\Psi$ and intensity of light scattering $I$ are linear combinations of $\varphi_{1}$ and $\varphi_{2}$ :

$$
\begin{gathered}
\Psi=\lambda_{1} \varphi_{1}+\lambda_{2} \varphi_{2}+\Psi^{\mathrm{r}}, \\
I=\lambda_{1} \varphi_{1}+\lambda_{2} \varphi_{2}+I^{\mathrm{r}},
\end{gathered}
$$

where $\lambda_{i}$ are constants that depend on both the system and experimental arrangement, and $\Psi^{\mathrm{r}}$ and $I^{\mathrm{r}}$ are the background (regular) parts of $\Psi$ and $I$, respectively. According to the experiments [5], the optical activity and intensity of light scattering behave very much like the entropy predicted by Eq. (27).

\section{HEAT CAPACITY MEASUREMENTS}

The phase diagram for chiral-racemic mixtures of $S, S$-MBBPC [5] is presented in Fig. 1, where the paths of constant chiral fraction used for the heat capacity measurements are shown. The mixture with chiral fraction $X=0.45$ is the lowest chiral fraction mixture with no evidence for a two phase region.

With four unknown mixing coefficients and two unknown constants to describe a linear background, a general fit of the theory to the experimental heat capacity data involves six fitting parameters. However, $a_{1}$ can be set to zero since the slope of the $\mathrm{BP}_{\mathrm{III}}$ ISO transition line in the temperaturechiral fraction plane is very small. In addition, it is convenient to make some substitutions for $\varphi_{1}$ (mean field theory) and $r$ (scaling theory), so that some fitting parameters do not appear in the equation of state, and some fitting parameters appear grouped together in the expression for the heat capacity.

For mean field theory, by defining a normalized order parameter

$$
\hat{\varphi}_{1}=\sqrt{\frac{u_{0}}{a_{0} b_{1}}} \varphi_{1}
$$

the cubic equation to be solved becomes

$$
\hat{\varphi}_{1}^{3}+\hat{h}_{2} \hat{\varphi}_{1}-\hat{h}_{1}=0,
$$

where

$$
\begin{gathered}
\hat{h}_{1}=\sqrt{\frac{u_{0}}{a_{0}^{3} b_{1}^{3}}} h_{1}=\hat{a}_{1} \Delta X+\hat{a}_{2} \Delta T, \\
\hat{h}_{2}=\frac{h_{2}}{b_{1}}=\Delta T+\hat{b}_{2} \Delta X,
\end{gathered}
$$

with

$\hat{a}_{1}=\sqrt{\frac{u_{0}}{a_{0}^{3} b_{1}^{3}}} a_{1}$ and $\hat{a}_{2}=\sqrt{\frac{u_{0}}{a_{0}^{3} b_{1}^{3}}} a_{2}$ and $\hat{b}_{2}=b_{2} / b_{1}$.

The heat capacity expression then becomes

$$
C_{P, X}=\frac{R T}{T_{\mathrm{c}}} \frac{\left(\hat{a}_{2}-\hat{\varphi}_{1}\right)^{2}}{\hat{u}_{0}\left[\Delta T+\hat{b}_{2} \Delta X+3 \hat{\varphi}_{1}^{3}\right]}+A T_{\mathrm{c}} \Delta T+B,
$$

which involves only 5 fitting parameters $\hat{a}_{2}, \hat{b}_{2} \Delta X, A, B$, and $\hat{u}_{0}=u_{0} / a_{0}^{2} b_{1}^{2}$, if the parameter $a_{1}$ is set to zero.

For scaling theory, by defining a new parametric variable

$$
\hat{r}=\frac{r}{b_{1}},
$$

the equation of state is now given by

$$
\begin{gathered}
\hat{r}^{\beta+\gamma} \theta\left(1-\theta^{2}\right)=\hat{a}_{1} \Delta X+\hat{a}_{2} \Delta T, \\
\hat{r}\left(1-b^{2} \theta^{2}\right)=\Delta T+\hat{b}_{2} \Delta X,
\end{gathered}
$$

where

$$
\hat{a}_{1}=\frac{a_{1}}{a b_{1}^{\beta+\gamma}} \quad \text { and } \quad \hat{a}_{2}=\frac{a_{2}}{a b_{1}^{\beta+\gamma}} \quad \text { and } \quad \hat{b}_{2}=\frac{b_{2}}{b_{1}} \text {. }
$$

The expression for the heat capacity is

$$
\begin{aligned}
C_{P, X}= & \frac{R T}{T_{\mathrm{c}}}\left(a k b_{1}^{2 \beta+\gamma}\right)\left[\left(\hat{a}_{2}\right)^{2} \hat{r}^{-\gamma} c_{1}(\theta)+\hat{r}^{-\alpha} c_{2}(\theta)\right. \\
& \left.+2\left(\hat{a}_{2}\right) \hat{r}^{\beta-1} c_{12}(\theta)\right]+A T_{\mathrm{c}} \Delta T+B,
\end{aligned}
$$

which again involves only 5 fitting parameters if $a_{1}=0$. It should be pointed out that the last term in expression (51) for $\chi_{2}$ is included in the background heat capacity.

Least square fits to the $X=0.45$ heat capacity data were performed for both equations of state. Since $X_{\mathrm{c}}$ is unknown, only $\hat{b}_{2} \Delta X$ is determined by these fits. As explained later, however, by also performing fits to the heat capacity data taken at other values of $X$, we are able to determine $X_{\mathrm{c}}$ and calculate values for $\hat{b}_{2}$ and $\Delta X$. Values for $\hat{b}_{2}$ and $\Delta X$ are therefore given in the tables. Because a small amount of modulation $( \pm 17 \mathrm{mK})$ was present during the heat capacity measurements, the fitting procedure utilized heat capacity expressions modified by this amount of modulation. The values for the fitting coefficients are shown in Table II and graphs of the theoretical fits and experimental data are shown in Fig. 5. Notice that the fit using scaling theory is significantly better than the fit using mean field theory (the reduced 
TABLE II. Heat capacity fitting parameters for $S, S$-MBBPC $(X=0.45)$. The units of the background slope and background at $T_{\mathrm{c}}$ are $\mathrm{J} /\left(\right.$ mole $\left.\mathrm{K}^{2}\right), \mathrm{J} /($ mole $\mathrm{K})$, respectively.

\begin{tabular}{lcc}
\hline \hline & Mean field theory & Scaling theory \\
\hline$T_{\mathrm{c}}$ & $392.484 \mathrm{~K}$ & $392.485 \mathrm{~K}$ \\
$\Delta X$ & $0.15 \pm 0.10$ & $0.18 \pm 0.08$ \\
$\hat{u}_{0}^{-1}$ or $a k b_{1}^{2 \beta+\gamma}$ & $13.53 \pm 0.16$ & $3.836 \pm 0.034$ \\
$\hat{a}_{2}\left(\times 10^{-2}\right)$ & $-8.324 \pm 0.079$ & $-6.206 \pm 0.050$ \\
$\hat{b}_{2}\left(\times 10^{-3}\right)$ & $2.2 \pm 1.5$ & $2.2 \pm 1.0$ \\
Background slope & $220.5 \pm 6.5$ & $79.4 \pm 4.7$ \\
Background at $T_{\mathrm{c}}$ & $747.4 \pm 1.8$ & $787.3 \pm 1.5$ \\
Reduced chi square & 41 & 26 \\
\hline \hline
\end{tabular}

chi squares is smaller). These fits were in fact quite stable, in that fitting data over a larger range or eliminating data near $T_{c}$ (in case impurity effects are important) changed the values of the fitting parameters by amounts roughly equal to their uncertainties.

Fits to the heat capacity data for other values of $X$, both above and below $X=0.45$, were also performed. In these cases $\hat{a}_{2}$ was fixed at the value found for the $X=0.45$ mixture while $\hat{b}_{2} \Delta X$ remained a fitting parameter. The results are shown in Fig. 6 for the $X=0.40$ mixture and in Fig. 7 for the $X=0.50$ mixture. The scaling theory fits are again better than when mean field theory is used. It should be pointed out that there are problems with fitting the $X=0.40$ data. The slight "bump" on the low-temperature branch of the data is due to the $\mathrm{BP}_{\mathrm{I}^{-}}-\mathrm{BP}_{\mathrm{III}}$ phase transition, and the excess heat capacity due to this transition has not been taken into account. Additionally, there is some question whether all the data used were truly static heat capacity data, as the data very

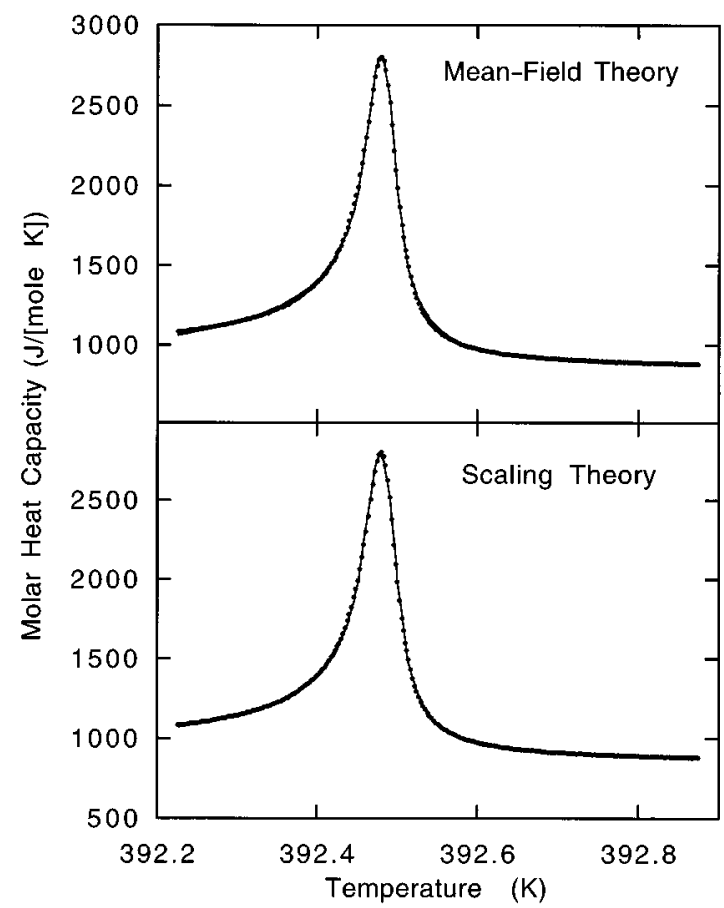

FIG. 5. Molar heat capacity data from Ref. [5] and fits (solid lines) for $X=0.45$. close to $T_{c}$ still showed a frequency dependence. However, the analysis of the $X=0.40$ data is completely consistent with the results for the other mixtures, which do not suffer from these problems, so it is included for completeness. When the results from the $X=0.40,0.45$, and 0.50 fits plus similar fits for the $X=0.55$ and $X=0.75$ mixtures (Figs. 8 and 9) are all combined, the estimates for $X_{c}$ are 0.39 \pm 0.04 from the mean field theory fits and $0.38 \pm 0.03$ from the scaling theory fits (Fig. 10). The values of the fitting parameters for the four additional mixtures are given in Table III. When $F$ tests are applied between the mean field and scaling theory fits, the difference in the quality of the fits is significant at the $99 \%$ confidence level in all cases except $X=0.75$, where the difference is significant at the $93 \%$ confidence level.

\section{OTHER MEASUREMENTS}

Optical activity and light scattering measurements have also been performed on the $X=0.45$ mixture $[5,32]$. Actually, a turbidity experiment with right-circularly polarized light was done in place of a light scattering experiment, but turbidity should be proportional to the total cross section for light scattering. Fits were performed assuming that both of these properties depend on a linear combination of the two scaling densities and fixing the values of $\hat{a}_{2}$ and $\hat{b}_{2} \Delta X$ at the values determined by the $X=0.45$ heat capacity fit. For the mean field theory fits, the equation is

$$
\Psi=\lambda_{1} \sqrt{\frac{a_{0} b_{1}}{u_{0}}} \hat{\varphi}_{1}-\frac{1}{2} \lambda_{2} \frac{a_{0}^{2} b_{1}}{u_{0}} \hat{\varphi}_{1}^{2}+A T_{\mathrm{c}} \Delta T+B
$$

and for scaling theory fits, the equation is

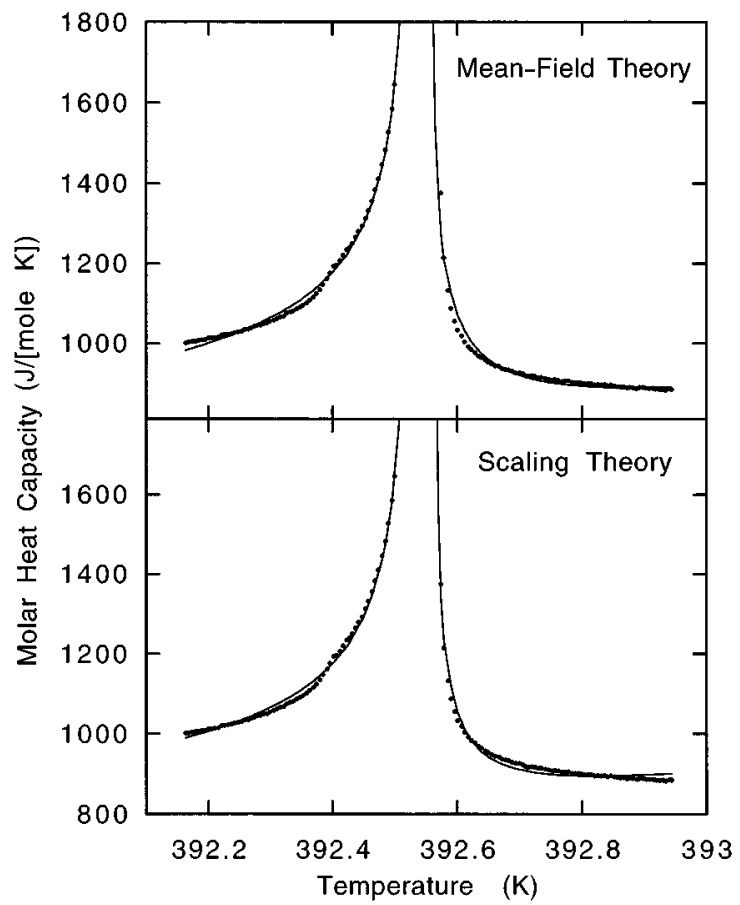

FIG. 6. Molar heat capacity data from Ref. [5] and fits (solid lines) for $X=0.40$. 


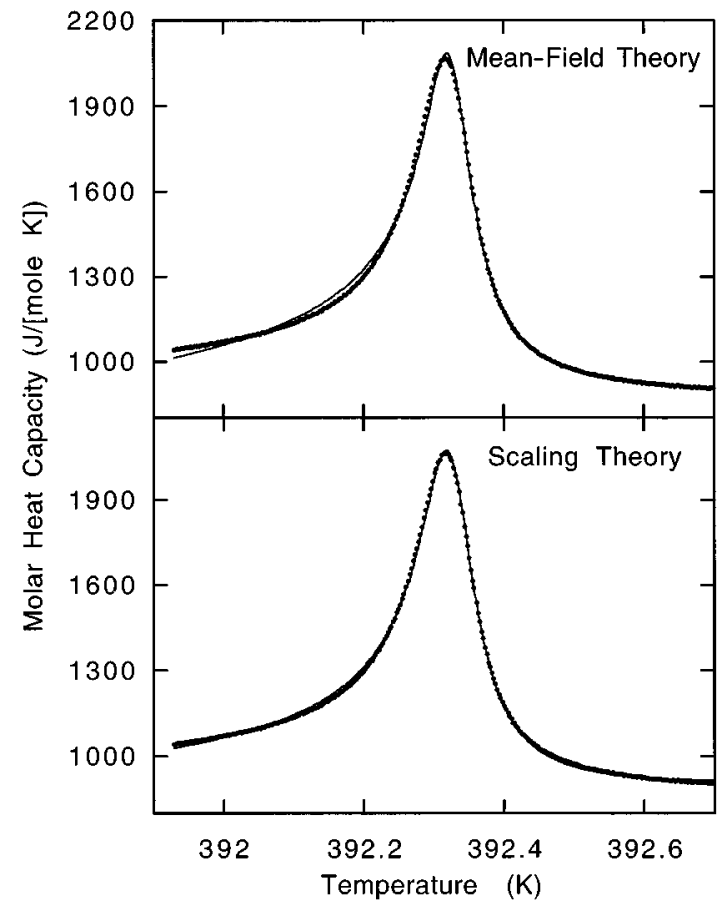

FIG. 7. Molar heat capacity data from Ref. [5] and fits (solid lines) for $X=0.50$.

$$
\Psi=\lambda_{1} k b_{1}^{\beta} \hat{r}^{\beta} \theta+\lambda_{2} a k b_{1}^{1-\alpha} \hat{r}^{1-\alpha} s(\theta)+A T_{\mathrm{c}} \Delta T+B .
$$

In order to keep the number of fitting parameters to a minimum, the scaling theory equation omits the last term in expression (48) for $\varphi_{2}$. This term contributes a relatively small amount and varies slowly with temperature, so it is approxi-

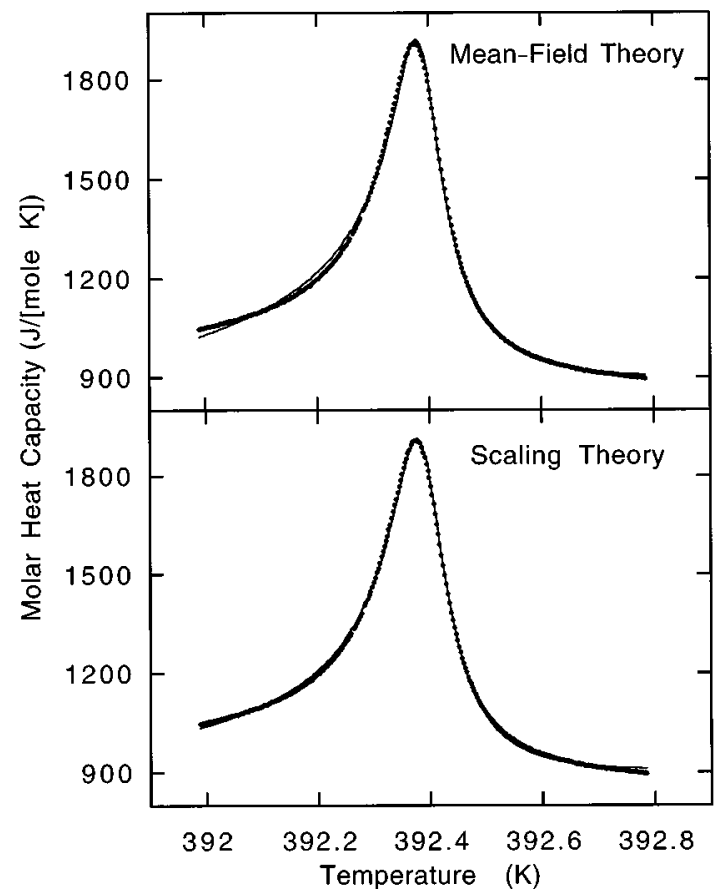

FIG. 8. Molar heat capacity data from Ref. [5] and fits (solid lines) for $X=0.55$.

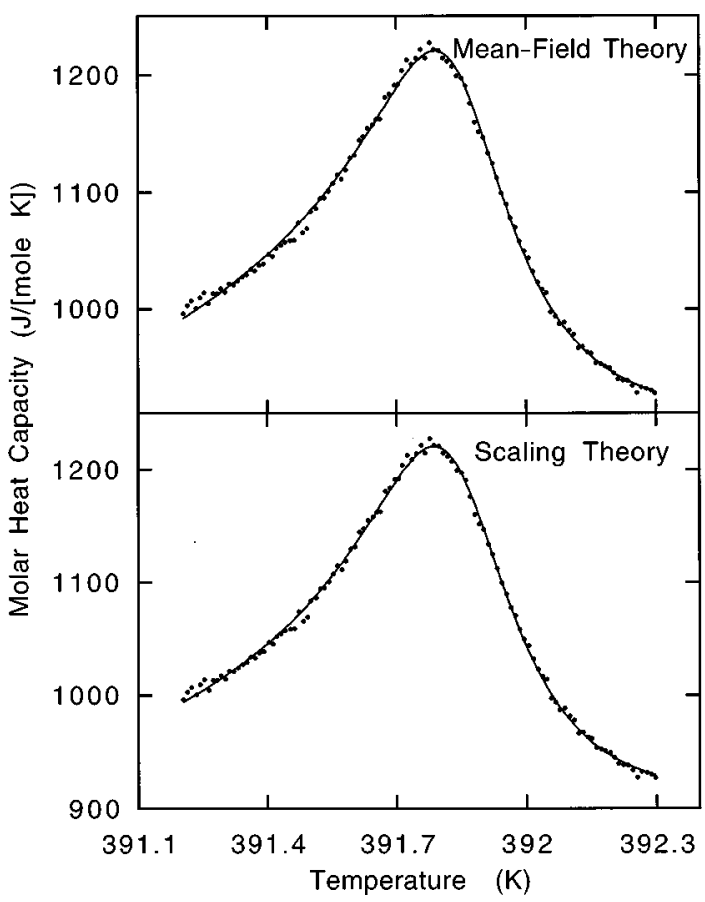

FIG. 9. Molar heat capacity data from Ref. [5] and fits (solid lines) for $X=0.75$.

mated by the linear background terms, which were included to account for the regular contribution. Similar expressions were used to fit the turbidity data. Only a constant background term was included in the turbidity fits, since the fits were little improved by inclusion of a term with nonzero slope. The results are given in Table IV and Fig. 11. As with the heat capacity fits, the fits using scaling theory are equal to or better than the fits using mean field theory.

Latent heat measurements on the S-CE4 system indicate that the $X=1.0$ mixture is very close to the critical chiral fraction [13]. Optical activity and light scattering data [33] also exist on the $X=1.0$ mixture. Since the latent heat data are not precise enough to determine $\hat{a}_{2}, \hat{b}_{2}$, and $X_{C}$ accurately, values for these three parameters were selected so that good fits to both the thermodynamic and optical data result (see Table V and Figs. 12 and 13). The mean field equation for the latent heat fit is

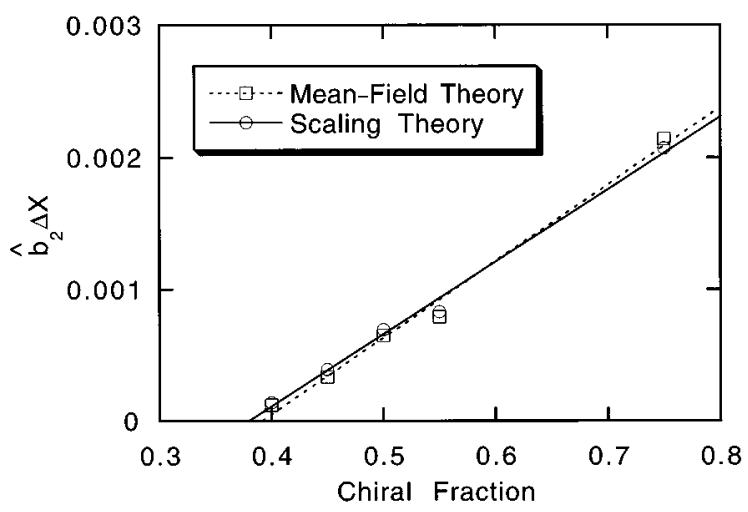

FIG. 10. Determination of the critical chiral fraction $X_{\mathrm{c}}$. 
TABLE III. Heat capacity fitting parameters for $S, S$-MBBPC mixtures based on the values of $\hat{a}_{2}$ from Table II. The units of the background slope and background at $T_{\mathrm{c}}$ are $\mathrm{J} /(\text { mole } \mathrm{K})^{2} \mathrm{~J} /($ mole $\mathrm{K})$, respectively.

\begin{tabular}{lcccc}
\hline \hline & \multicolumn{2}{c}{$X=0.40$} & \multicolumn{2}{c}{$X=0.50$} \\
& Mean field theory & Scaling theory & Mean field theory & Scaling theory \\
\hline$T_{\mathrm{c}}$ & $392.540 \mathrm{~K}$ & $392.544 \mathrm{~K}$ & $392.331 \mathrm{~K}$ & $392.334 \mathrm{~K}$ \\
$\Delta X$ & $\sim 0.02$ & $\sim 0.05$ & $0.28 \pm 0.10$ & $0.32 \pm 0.09$ \\
$\hat{u}_{0}^{-1}$ or $a k b_{1}^{2 \beta+\gamma}$ & $11.49 \pm 0.15$ & $4.046 \pm 0.043$ & $14.361 \pm 0.067$ & $4.226 \pm 0.012$ \\
$\hat{b}_{2}\left(\times 10^{-3}\right)$ & & & $2.3 \pm 0.8$ & $2.2 \pm 0.6$ \\
Background slope & $155.0 \pm 7.9$ & $140.7 \pm 6.1$ & $193.0 \pm 4.4$ & $123.1 \pm 2.6$ \\
Background at $T_{\mathrm{c}}$ & $783.1 \pm 2.7$ & $771.3 \pm 2.3$ & $765.3 \pm 1.9$ & $780.4 \pm 1.1$ \\
Reduced chi square & 33 & 21 & 14 & 5 \\
& & & & \\
& & $X=0.55$ & & \\
& & & & \\
& & & & \\
$T_{\mathrm{c}}$ & & & & \\
$\hat{u}_{0}^{-1}$ or $a k b_{1}^{2 \beta+\gamma}$ & $15.030 \pm 0.067$ & $4.4821 \pm 0.0014$ & $12.49 \pm 0.12$ & $3.832 \pm 0.028$ \\
$\hat{b}_{2}\left(\times 10^{-3}\right)$ & $1.9 \pm 0.5$ & $1.8 \pm 0.4$ & $2.3 \pm 0.4$ & $2.1 \pm 0.2$ \\
Background slope & $187.9 \pm 3.7$ & $122.6 \pm 2.6$ & $82.0 \pm 2.2$ & $60.7 \pm 1.8$ \\
Background at $T_{\mathrm{c}}$ & $758.6 \pm 1.8$ & $767.1 \pm 1.2$ & $817.0 \pm 1.8$ & $804.1 \pm 1.5$ \\
Reduced chi square & 35 & 18 & 1.2 & 0.9 \\
\hline \hline
\end{tabular}

$$
L=2 \frac{a_{0}^{2} b_{1}^{2}}{u_{0}} R T_{\mathrm{c}} \hat{a}_{2} \hat{\varphi}_{1}
$$

and the scaling equation is

$$
L=2 a k b_{1}^{2 \beta+\gamma} R T_{\mathrm{c}} \hat{a}_{2} \hat{r}^{\beta} \theta
$$

No background terms were included in the fits to the optical activity data and only a constant value for the background contribution was included in the fits to the light scattering data, since the inclusion of additional terms did not make the fits significantly better. Interestingly, the sign of the $\varphi_{2}$ contribution to the light scattering is opposite from that for $S, S$-MBBPC. Note that these fits suggest that the $X=1.0$ mixture is slightly above the critical point even though the latent heat data indicate that it is slightly below the critical point [13]. The choice of $X_{c}=0.95$ is necessary in order to achieve good fits not only to the latent heat data, but to the optical activity and light scattering data as well. We include the data on S-CE4 simply to show that a reasonable selection of parameters is capable of describing both the thermodynamic and optical data in this system also.

\section{DISCUSSION}

The fact that both the mean field and scaling theories describe both the thermodynamic and optical data not just near the critical point but across the phase diagram is strong evidence that the $\mathrm{BP}_{\mathrm{III}}$ ISO transition is analogous to the liquid-gas transition. The fact that the scaling theory is better in its agreement with the experimental data also is strong evidence that this transition belongs to the same universality class as the 3D Ising model.

Just as interesting is the fact that the physical variables mix quite differently than in the liquid-gas transition. With $a_{1} \approx 0, h_{1}$ is nearly temperature, making the ordering field a temperature instead of a chemical potential (for the liquidgas transition) or magnetic field (for the magnetic transition). Instead of the order parameter being density or magnetization, the order parameter for the $\mathrm{BP}_{\mathrm{III}}-\mathrm{ISO}$ transition is

TABLE IV. Optical fitting parameters for $S, S$-MBBPC $(X=0.45)$. The units for the optical activity parameters are $\mathrm{rad} / \mathrm{cm}$, except for the background slope, which is $\mathrm{rad} /(\mathrm{cm} \mathrm{K})$. The units for the turbidity parameters are $\mathrm{cm}^{-1}$, except for the background slope which is $(\mathrm{cm} \mathrm{K})^{-1}$.

\begin{tabular}{lcccc}
\hline \hline & \multicolumn{2}{c}{ Optical activity } & \multicolumn{2}{c}{ Turbidity } \\
& Mean field theory & Scaling theory & Mean field theory & Scaling theory \\
\hline$T_{\mathrm{c}}$ & $390.915 \mathrm{~K}$ & $390.920 \mathrm{~K}$ & $391.025 \mathrm{~K}$ & $391.031 \mathrm{~K}$ \\
$\lambda_{1} \sqrt{a_{0} b_{1} / u_{0}}$ or $\lambda_{1} k b_{1}^{\beta}$ & $-44.7 \pm 1.4$ & $-7.69 \pm 0.30$ & $104.6 \pm 1.7$ & $30.04 \pm 0.21$ \\
$\lambda_{2} a_{0}^{2} b_{1} / u_{0}$ or $\lambda_{2} a k b_{1}^{1-\alpha}$ & $3340 \pm 59$ & $979 \pm 16$ & $854 \pm 147$ & $153.9 \pm 9.6$ \\
Background slope & $2.00 \pm 0.29$ & $-1.58 \pm 0.25$ & 0 (fixed) & 0 (fixed) \\
Background at $T_{\mathrm{c}}$ & $-0.039 \pm 0.013$ & $-0.925 \pm 0.009$ & $4.575 \pm 0.085$ & $4.096 \pm 0.012$ \\
Reduced chi square & 0.7 & 0.8 & 30 & 1.8 \\
\hline \hline
\end{tabular}




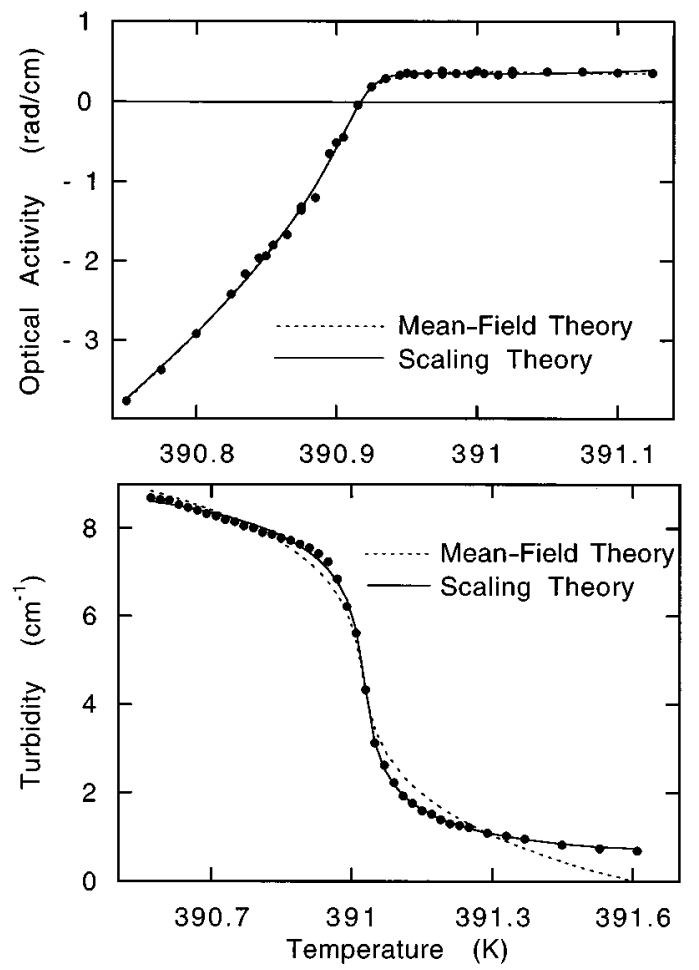

FIG. 11. Optical data and fits for the $X=0.45$ mixture.

closely related to entropy. In fact, the smallness of $b_{2}$ demonstrates that temperature is the most important contribution to both the ordering and nonordering fields. Further theoretical work will be required to clarify a connection between the order parameter $\varphi_{1}$ and the chiral order parameter introduced by Lubensky and Stark [7], as well as a microscopic interpretation for the mixing coefficients.

TABLE V. Thermodynamic and optical fitting parameters for S-CE4 based on $X_{c}=0.95$ and $\hat{a}_{2}=-0.2, \hat{b}_{2}=0.01$ (mean field theory) or $\hat{a}_{2}=-0.06, \hat{b}_{2}=0.003$ (scaling theory).

Latent heat

Mean field theory Scaling theory

\begin{tabular}{lll}
\hline$a_{0}^{2} b_{1}^{2} / u_{0}$ or $a k b_{1}^{2 \beta+\gamma}$ & 1.1 & 5.0 \\
Reduced chi square & 0.7 & 0.9
\end{tabular}

\begin{tabular}{lcc}
\hline & \multicolumn{2}{c}{ Optical activity } \\
& Mean field theory & Scaling theory \\
\hline$T_{\mathrm{c}}$ & $310.34 \mathrm{~K}$ & $310.35 \mathrm{~K}$ \\
$\lambda_{1} \sqrt{a_{0} b_{1} / u_{0}}$ or $\lambda_{1} k b_{1}^{\beta}$ & $-42 \mathrm{rad} / \mathrm{cm}$ & $-5.4 \mathrm{rad} / \mathrm{cm}$ \\
$\lambda_{2} a_{0}^{2} b_{1} / u_{0}$ or $\lambda_{2} a k b_{1}^{1-\alpha}$ & $1600 \mathrm{rad} / \mathrm{cm}$ & $910 \mathrm{rad} / \mathrm{cm}$ \\
Reduced chi square & 1.0 & 2.8 \\
\hline \multicolumn{2}{c}{ Light scattering } \\
& Mean field theory & Scaling theory \\
\hline$T_{\mathrm{c}}$ & $310.21 \mathrm{~K}$ & $310.21 \mathrm{~K}$ \\
$\lambda_{1} \sqrt{a_{0} b_{1} u_{0}}$ or $\lambda_{1} k b_{1}^{\beta}$ & $130 \mathrm{kcounts} / \mathrm{s}$ & $22 \mathrm{kcounts} / \mathrm{s}$ \\
$\lambda_{2} a_{0}^{2} b_{1} / u_{0}$ or $\lambda_{2} a k b_{1}^{1-\alpha}$ & $-3700 \mathrm{kcounts} / \mathrm{s}$ & $-2000 \mathrm{kcounts} / \mathrm{s}$ \\
Background at $T_{c}$ & $4.1 \mathrm{kcounts} / \mathrm{s}$ & $4.3 \mathrm{kcounts} / \mathrm{s}$ \\
Reduced chi square & 0.6 & 0.6 \\
\hline \hline
\end{tabular}

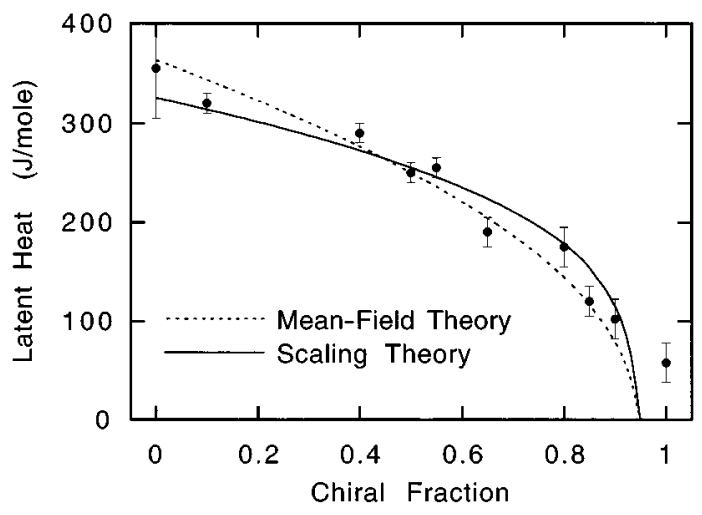

FIG. 12. Latent heat data and fits for S-CE4. $X_{\mathrm{c}}=0.95, \hat{a}_{1}=0$, and $\hat{a}_{2}=-0.2, \hat{b}_{2}=0.01$ (mean field theory) or $\hat{a}_{2}=-0.06, \hat{b}_{2}$ $=0.003$ (scaling theory).

The variation of $\Delta X$ with chiral fraction in $S, S$-MBBPC suggests that the critical chiral fraction $X_{\mathrm{c}}$ is slightly below $X=0.40$. However, the heat capacity measurements on the $X=0.40$ mixture showed (1) a difference between the ac calorimetric and nonadiabatic scanning results and (2) characteristic changes in the phase shift between the heater power and the observed sample temperature, which normally provide strong evidence for a two-phase coexistence at a discontinuous transition. As stated above, fitting the $X$ $=0.40$ data was problematic and it is included in the analysis for completeness, but it is also true that the fits to the other heat capacity data are consistent with a critical point around $X=0.40$. We have tested the possibility that our method of analysis overemphasizes supercriticality, but have found no
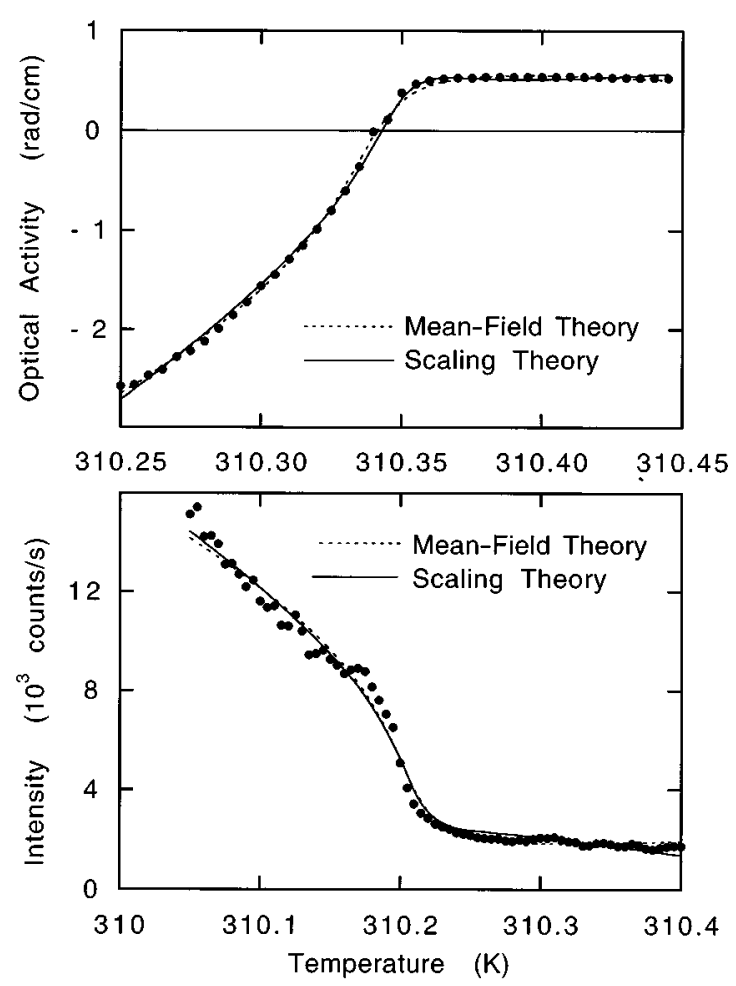

FIG. 13. Optical data and fits for S-CE4. $X_{\mathrm{c}}=0.95, \hat{a}_{1}=0$, and $\hat{a}_{2}=-0.2, \hat{b}_{2}=0.01$ (mean field theory) or $\hat{a}_{2}=-0.06, \hat{b}_{2}$ $=0.003$ (scaling theory). 
clear indication that this is the case. It is interesting to note that the result of our analysis of the S-CE4 data is similar, in that the $X=1.0$ mixture gives experimental evidence for being below the critical point, while our analysis indicates that it is above the critical point. More accurate experiments are required to clarify this issue.

It should also be pointed out that we have included background terms to account for any noncritical contributions to the data. The heat capacity background contributions fall above the actual data, not in the region of the fit, but at higher temperatures. This is probably the result of the fitting procedure used, since including the higher temperature data changes the background contributions so they fall below all of the data, resulting in slightly different fitting parameters but a fit of the same quality.

\section{CONCLUSIONS}

The $\mathrm{BP}_{\mathrm{III}}$ ISO critical point is unique. Whereas the mixing of the physical fields is of minor importance in the liquid gas transition, which is rather symmetric near the critical point, this feature is crucial to understanding the nature of the $\mathrm{BP}_{\mathrm{III}}$-ISO transition. Whether this is true for other liquid crystal transitions, e.g., the smectic- $A-$ smectic- $A_{d}$ transition, is unclear. The important point is that whenever the physical fields are mixed, the system does not follow simple powerlaw behavior.

The critical slowing down of the order parameter of this transition may reveal itself in a dynamic light scattering experiment if there is coupling between the fluctuations of the order parameter and the refractive index fluctuations. Such an experiment was attempted, but was hampered by multiple scattering effects near the critical point [32]. Another experiment is planned for the near future. Perhaps more revealing would be an acoustic measurement, since sound couples to temperature, which clearly is the dominant variable in this system.

The $\mathrm{BP}_{\mathrm{III}}$-ISO critical point still remains the only experimentally observed example of a fluid-fluid critical point that is not associated with the liquid-gas transition or with the demixing transition in binary liquids. However, the recent modeling of liquid water predicts the coexistence of two aqueous phases in the supercooled metastable region [34]. One may expect finding other systems in which two fluid phases of the same symmetry but of different structure coexist, and the transition terminates at a critical point. The approach developed in this paper may be useful for a description of such phenomena.

\section{ACKNOWLEDGMENTS}

The research at the University of Maryland was supported by the Office of Basic Energy Sciences of the U.S. Department of Energy under Grant No. DE-FG02-95ER-14509. The research at Swarthmore College was supported by the National Science Foundation under Grant No. DMR9224617. The authors would especially like to thank G. Iannacchione for providing the heat capacity data. Useful discussions with M. Fisher, C. Garland, P. Keyes, T. Lubensky, J. Sengers, H. Stark, and J. Thoen are gratefully acknowledged.
[1] P. G. de Gennes and J. Prost, The Physics of Liquid Crystals (Clarendon, Oxford, 1995).

[2] E. P. Koistinen and P. H. Keyes, Phys. Rev. Lett. 74, 4460 (1995).

[3] T. Seideman, Rep. Prog. Phys. 53, 659 (1990); D. C. Wright and N. D. Mermin, Rev. Mod. Phys. 61, 385 (1989); P. P. Crooker, Liq. Cryst. 5, 751 (1989); R. M. Horneich and S. Shtrikman, Mol. Cryst. Liq. Cryst. 165, 183 (1989); V. A. Belyakov and V. E. Dmitrienko, Usp. Fiz. Fiz. Nauk 146, 369 (1985) [ Sov. Phys. Usp. 28, 535 (1985)].

[4] Z. Kutnjak, C. W. Garland, J. L. Passmore, and P. J. Collings Phys. Rev. Lett. 74, 4859 (1995).

[5] Z. Kutnjak, C. W. Garland, C. G. Schatz, P. J. Collings, C. J. Booth, and J. W. Goodby, Phys. Rev. E 53, 4955 (1996); The absolute temperatures used here for the heat capacity data differ slightly from those reported previously to account for an additive calibration error in the previously published data [C. W. Garland (private communication)].

[6] H. Stegemeyer and K. Bergmann, in Liquid Crystals of Oneand Two-Dimensional Order, edited by W. Helfrich and G. Heppke (Springer-Verlag, Berlin, 1980), p. 161.

[7] T. C. Lubensky and H. Stark, Phys. Rev. E 53, 714 (1996).

[8] N. D. Mermin and J. J. Rehr, Phys. Rev. Lett. 26, 1155 (1971).

[9] A. Z. Patashinskii and V. L. Pokrovskii, Fluctuation Theory of Phase Transitions (Pergamon, Oxford, 1979).
[10] M. A. Anisimov, E. E. Gorodetskii, V. D. Kulikov, and J. V. Sengers, Phys. Rev. E 51, 1199 (1995).

[11] M. A. Anisimov, A. A. Povodyrev, E. E. Gorodetskii, V D. Kulikov, and J. V. Sengers, Physica A 220, 277 (1996).

[12] J. V. Sengers and J. M. H. Levelt Sengers, Annu. Rev. Phys. Chem. 37, 189 (1986).

[13] G. Voets, Ph.D. thesis, University of Leuven, Belgium, 1992 (unpublished).

[14] B. Widom, J. Chem. Phys. 43, 2998 (1965).

[15] L. P. Kadanoff, Physics (Long Island City, NY) 2, 263 (1966)

[16] J. M. H. Levelt Sengers, R. J. Hocken, and J. V. Sengers, Phys. Today 30(12), 42 (1977).

[17] J. V. Sengers and J. M. H. Levelt Sengers, Progress in Liquid Physics (Wiley, Chichester, U.K., 1978), Chap. 4.

[18] Phase Transitions: Cargèse 1980, edited by M. Levy, J. C. Le Guillou, and J. Zinn-Justin (Plenum, New York, 1981).

[19] M. E. Fisher, in Critical Phenomena, edited by F. J. W. Hahne, Lecture Notes in Physics Vol. 186 (Springer, Berlin, 1982), p. 1.

[20] M. A. Anisimov, Critical Phenomena in Liquids and Liquid Crystals (Gordon and Breach, Philadelphia, 1991).

[21] A. J. Liu and M. E. Fisher, Physica A 156, 35 (1989).

[22] S. B. Kiselev and J. V. Sengers, Int. J. Thermophys. 14, 1 (1993).

[23] M. Ley-Koo and M. S. Green, Phys. Rev. A 16, 2483 (1977). 
[24] P. Schofield, Phys. Rev. Lett. 22, 606 (1969).

[25] B. D. Josephson, J. Phys. C 2, 1113 (1969).

[26] J. T. Ho and J. D. Litster, Phys. Rev. Lett. 22, 603 (1969).

[27] J. T. Ho and J. D. Litster, Phys. Rev. B 2, 4532 (1970).

[28] P. Schofield, J. D. Litster, and J. T. Ho, Phys. Rev. Lett. 23, 1098 (1969).

[29] M. A. Anisimov, S. B. Kiselev, J. V. Sengers, and S. Tang, Physica A 188, 487 (1992).
[30] K. G. Wilson, Phys. Rev. Lett. 28, 548 (1972).

[31] K. G. Wilson and J. Kogut, Phys. Rep. 12C, 77 (1974).

[32] U. Singh, P. J. Collings, C. J. Booth, and J. W. Goodby, J. Phys. II 7, 1683 (1997).

[33] J. B. Becker and P. J. Collings, Mol. Cryst. Liq. Cryst. 265, 163 (1995).

[34] P. G. Debenedetti, Metastable Liquids: Concepts and Principles (Princeton University Press, Princeton, NJ, 1996). 